\title{
Historia natural del ecosistema de sabana del Valle del Río General, Costa Rica
}

\author{
Natural history of the savannah scosystem of the \\ General River Valley, Costa Rica
}

\author{
Guillermo Artavia* \\ Gerardo Ávalos*
}

Fecha de recibido: 19 de febrero e 2020

Fecha de aceptado: 20 de noviembre de 2020

\section{Resumen}

El ecosistema de sabana ocupa una importante superficie a nivel mundial con alrededor de 15 millones de $\mathrm{km}^{2}, 43 \%$ del área continental. En Costa Rica, la mayor extensión de sabana se encuentra en la cuenca del Río General en el sur del país. En este artículo analizamos el contexto biofísico, la historia paleoecológica y paleoclimática, el poblamiento humano pre-colombino, la exploración científica, y el efecto del fuego en el ecosistema de sabana. Estos aspectos han sido poco estudiados en las sabanas de Costa Rica, un ecosistema para el cual no se tiene claro su origen y evolución. Se realizó una exhaustiva revisión y análisis de la literatura sobre las sabanas del sur de Costa Rica. Se hizo una campaña de campo para verificar la distribución del ecosistema y explorar su estado de conservación. Las sabanas se desarrollan sobre una compleja heterogeneidad geológica y geomorfológica. Este ecosistema se encuentra amenazado por diferentes factores, principalmente la expansión agrícola, la fragmentación y la pérdida de hábitat. El origen de las sabanas, si son naturales o antrópicas, continúa siendo controversial. Lo que es claro es que es un ecosistema amenazado y con necesidad de integrar más esfuerzos para su uso científico y de conservación.

Palabras claves: ecosistema de sabana, Valle del Río General, historia natural.

* Escuela de Geografía. Universidad de Costa Rica, Costa Rica, correo electrónico: luis.artavia@ucr.ac.cr

* Escuela de Biología, Universidad de Costa Rica, Costa Rica, correo electrónico: gerardo.avalos@ucr.ac.cr 


\section{Abstract}

The savanna ecosystem covers an important surface worldwide with around 15 million $\mathrm{km}^{2}$, or $43 \%$ of the continental area. In Costa Rica, the largest expanse of savannas is found in the basin of Río General in southern Costa Rica. In this article we analyze the biophysical context, the paleoecological and paleoclimatic history, the pre-Columbian human settlement, the scientific exploration, and the effect of fire on the savanna ecosystem. These aspects have been poorly explored in the savannas of Costa Rica, an ecosystem whose origin and evolution is still not clear. An exhaustive review and analysis of the literature on the savannas of southern Costa Rica was carried out. A field campaign was carried out to verify the distribution of the ecosystem and explore its conservation status. Savannas develop over complex geological and geomorphological gradients. This ecosystem is threatened by different factors, mainly agricultural expansion, habitat fragmentation, and habitat loss. The origin of the savannas, whether they are natural or anthropic, continues to be controversial. What is clear is that it is a threatened ecosystem where more integrated efforts are necessary for its scientific and conservation use.

Key words: Savanna ecosystem, General River Valley, Natural History.

\section{Introducción}

La sabana es una compleja asociación de especies que interactúan a través de diferentes escalas espaciales y temporales (Lomolino et al., 2006). En sentido estricto, las sabanas se definen como un ecosistema con un estrato casi continuo de pastos, con árboles y arbustos dispersos, presentes en diferentes densidades, y en donde los patrones de crecimiento de la vegetación se encuentran claramente influidos por la estacionalidad climática, en particular la distribución de la precipitación, así como por factores edáficos e interacciones ecológicas, como por ejemplo la competencia por agua o nutrientes, así como por la influencia del fuego (Harris, 1980; Calabrese et al., 2010).

El término sabana tiene un origen español, inicialmente fue utilizado para referirse a praderas o llanuras, y posteriormente para describir diversos tipos de vegetación, desde ambientes en los que predomina una cubierta vegetal leñosa y áreas en las que se encuentran arbustos y árboles muy dispersos (Higgins et al., 2007). Harris (1980) indica que el vocablo se deriva de la palabra zabana, el cual es una adaptación al español del lenguaje de los indios arawak, nativos de las Grandes Antillas justo a la llegada de Cristóbal Colón. Vargas (2005) apunta que la palabra sabana fue empleada por primera vez por Gonzalo Fernández de Oviedo en 1535 para referirse a grandes extensiones de pastos en los llanos venezolanos.

La sabana es el tipo de vegetación más común que encontramos en los trópicos y subtrópicos. Cubren extensas áreas en América del Sur, África y Australia. Desde 
el punto de vista geográfico, las sabanas están ubicadas entre los $20^{\circ}$ de latitud $\mathrm{N} \mathrm{y}$ $\mathrm{S}$ en la región tropical. Este ecosistema cubre alrededor del $43 \%$ de la superficie terrestre, lo que equivale a unos 15 millones de $\mathrm{km}^{2}$ (Caylor y Shugart, 2006).

Las sabanas se originaron en el Eoceno, hace 55 millones de años, ya que los primeros registros de granos de polen de gramíneas en el trópico datan de la mitad de dicho periodo geológico (Van Der Hammen, 1993). Sin embargo, Beerling y Osborne (2006) proponen que esta formación vegetal más bien data del Mioceno tardío, hace unos 8 millones de años.

En Costa Rica, encontramos sabanas en la vertiente pacífica en la provincia de Guanacaste (Vargas, 2005), y en el interior del Valle del Río General (Sandner, 1962), y pequeñas áreas en el pacífico central, especialmente en el cantón de Turrubares, donde se encuentran las sabanas Rayo también conocidas como sabanas Turrubares (Figura 1). En el caso del Valle del General, se observan una serie de fragmentos de sabana de diferente tamaño, con vegetación propia de este ecosistema entre las localidades de Volcán y Boruca de Buenos Aires (Díaz, 2015). La primera referencia a estas sabanas en el Valle del Río General se remonta al año 1563, cuando fueron mencionadas por el conquistador español Antonio Álvarez Pereyra (Artavia, 2011). Otra evidencia histórica de la presencia de sabanas en el sur de Costa Rica se encuentra en el relato del conquistador Juan Vázquez de Coronado, quien en el año 1563 menciona la presencia de unas sabanas muy extensas en las inmediaciones de lo que hoy es Puerto Cortés, en ese momento conocido como El Pozo (Molina, 2005).

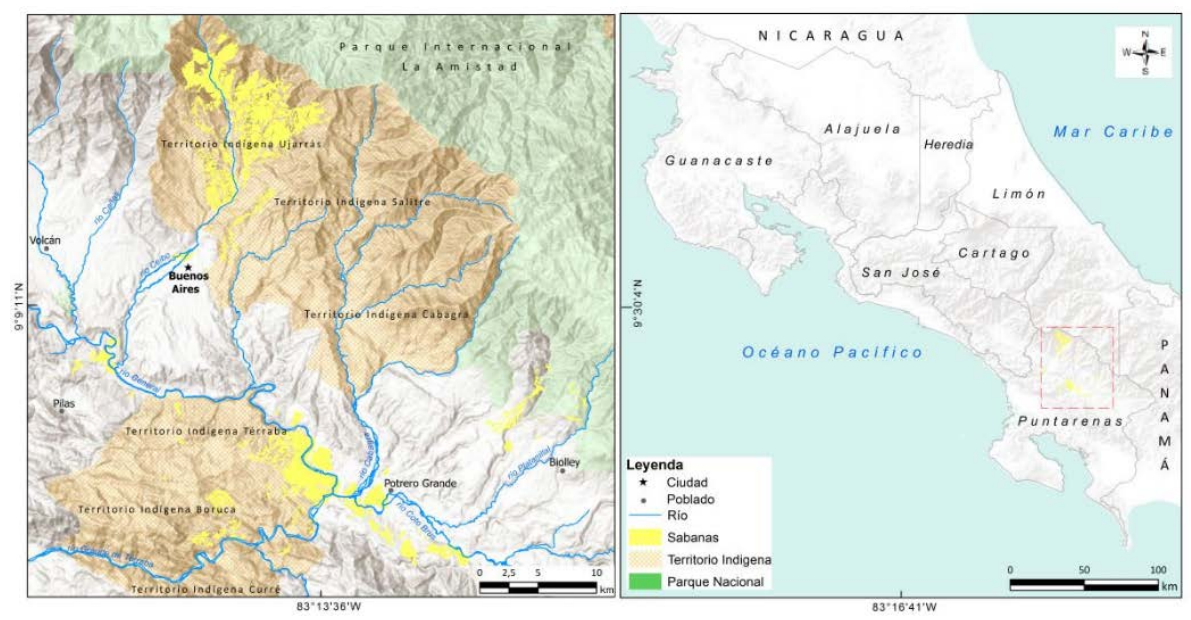

Figura 1. Mapa de distribución del ecosistema de sabana en el sur de Costa Rica. Fuente: elaboración propia con base en una imagen satelital RapidEye de cinco metros de resolución, 2013. 
El ecosistema de sabana ha sido poco estudiado. Su existencia se ha mencionado en una serie de documentos, pero sin profundizar en su análisis, y sobre todo sin tratar de esclarecer su origen. Esta falta de interés quizás obedece a la idea de que dicho ecosistema es de origen antropogénico, producto de un uso continuo del espacio por el ser humano, posiblemente desde los últimos 4 mil años (Haberland, 1961).

Sandner (1962) comparte la idea de Henri Pittier de que el área donde se ubican las sabanas en el sur de Costa Rica estuvo ampliamente poblada por indígenas desde tiempos remotos. Esta opinión sostiene que el ecosistema de sabana se originó, y ha logrado permanecer hasta hoy, debido a la ocupación humana, además de las condiciones climáticas y edáficas que favorecen su existencia.

En este artículo hacemos un análisis del ecosistema de sabana desde la óptica de su historia natural, abordando aspectos como los contextos biofísico, paleoclimático, paleoecológico, el poblamiento humano precolombino la exploración científica de este ecosistema a través de la historia, y la discusión del posible origen de la sabana.

\section{El ambiente físico del ecosistema}

En la zona sur de Costa Rica, el ecosistema de sabana se encuentra representado cartográficamente en las hojas topográficas: Buenos Aires, Chánguena, Cabagra, Coto Brus y General (Tabla 1). Sin embargo, algunas de las sabanas que fueron cartografiadas en estas hojas hoy en día muestran diferentes grados de alteración, o han desaparecido.

Tabla 1. Ubicación de las sabanas en las hojas topográficas, escala 1: 50000

\begin{tabular}{|c|c|}
\hline Hoja & Sabana \\
\hline Coto Brus & Palacios, Vueltas, Tablas \\
\hline Changuena & Comején, Bujera, Cuij Cuij, Cuasan, Mamey \\
\hline Cabagra & $\begin{array}{l}\text { Cola de Pato, Macho Montes, Térraba, Buril, Caracucha, } \\
\text { Helechales, Esperanza }\end{array}$ \\
\hline General & $\begin{array}{l}\text { Buenos Aires, Tigre, San Antonio, Cola de Pato, Kamankaua, } \\
\text { La Tinta, Concepción, Dibujada, Térraba, Bruran, Barranco, } \\
\text { San Joaquín, Calienta Tigre }\end{array}$ \\
\hline Buenos Aires & $\begin{array}{l}\text { Jakyaka, Bisuk, Oka, DitsiriSuk, Bekis, Palmital, Ujarras, } \\
\text { Santa María, Salitre, Sipar, Sebror, Achiote }\end{array}$ \\
\hline
\end{tabular}

Fuente: Instituto Geográfico Nacional, Costa Rica. 1975. 
Desde un punto de vista geológico, las sabanas se desarrollan sobre diferentes tipos de materiales, principalmente sobre rocas sedimentarias e ígneas (Tabla 2). Como se puede apreciar no existe una relación directa entre la presencia de las sabanas y el tipo de material geológico.

Cabe resaltar las sabanas localizadas dentro de la cuenca del río Ceibo (Figura 2), las cuales cuentan con una considerable extensión territorial. Estas sabanas se desarrollan sobre dos litologías, una compuesta por rocas intrusivas del Mioceno, y otra de formación más reciente, constituidas por depósitos aluvionales del Cuaternario. Las rocas intrusivas son dominantes dentro del área, y forman afloramientos en algunas sabanas. En las sabanas Ditsiri Suk y Santa María se han cartografiado las dos áreas más extensas de intrusiones graníticas identificadas hasta ahora en el país (Obando y Kussmaul, 2009). Estos mismos autores identificaron monzonitas y granito porfirítico con hormblenda, en las sabanas Santa María; granito con biotita en sabana Ditsiri Suk; andesitas en sabana Oka; mientras que en las sabanas Sipar y Salitre se han identificado basaltos y basaltos alcalinos relacionados en ambos casos con flujos piroclásticos; cornubionitas han sido localizadas en sabana Murur Bisuk, y finalmente, en la sabana del Cerro Cola de Dragón se han encontrado micro-granito, y muy cerca de ahí, en la confluencia entre el río Ceibo y el río Kuiyé, se ha identificado micro-monzodiorita cuarzosa con biotita. Las edades de estos materiales oscilan entre 9.5 y 9.85 millones de años (Ma) (Obando y Kussmaul, 2009).

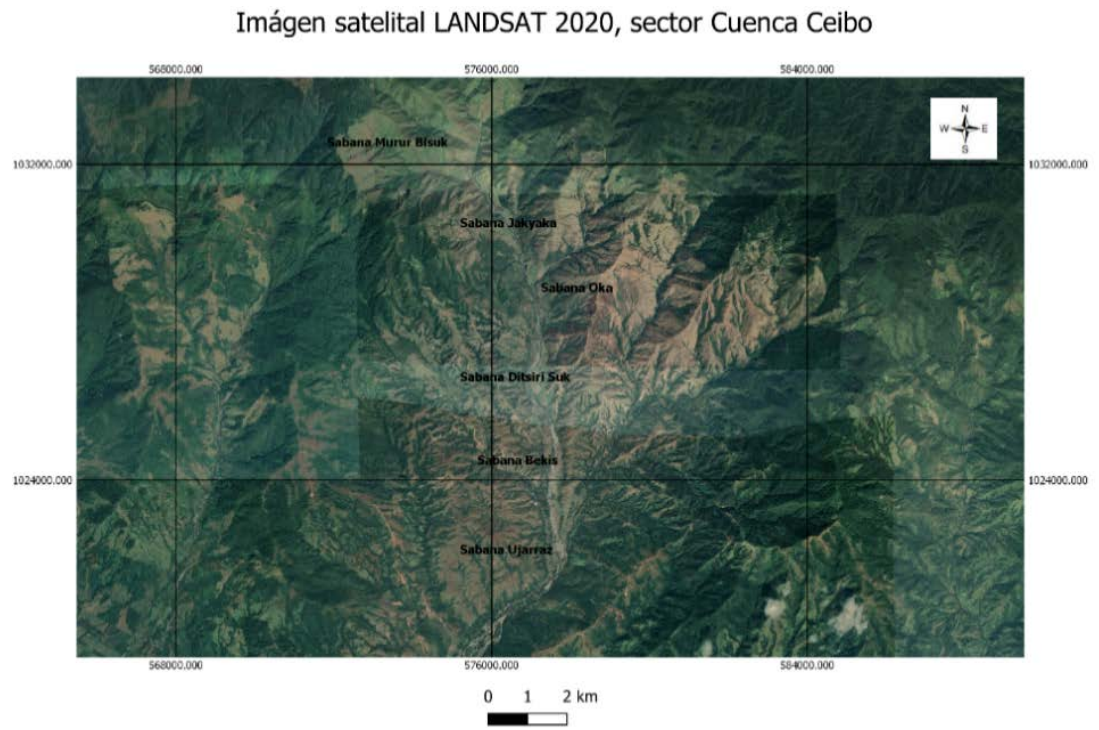

Figura 2. Área de sabanas en la cuenca hidrográfica del río Ceibo.

Fuente: elaboración propia, con base en una imagen satelital Landsat, 2020. 
Tabla 2. Materiales geológicos sobre los que se desarrollan algunas de las sabanas del sur de Costa Rica

\begin{tabular}{|c|c|}
\hline Sabana & Tipo de material \\
\hline Concepción & $\begin{array}{l}\text { Sedimentos aluviales y coluviales. Conglomerados y } \\
\text { areniscas aluviales (Formación Valle El General) }\end{array}$ \\
\hline $\begin{array}{l}\text { Dibujada, La Tinta, San Antonio, } \\
\text { Cola de Pato, El Tigre }\end{array}$ & $\begin{array}{l}\text { Conglomerados y areniscas aluviales (Formación } \\
\text { Valle El General) }\end{array}$ \\
\hline $\begin{array}{l}\text { Kamankaua, Térraba, Bruran, } \\
\text { Barranco }\end{array}$ & $\begin{array}{l}\text { Lavas, brechas y cuerpos hipoabisales: } 3.59 \text { a } 6.40 \\
\text { M.a. (Formación Grifo Alto). En el caso de la sabana } \\
\text { Térraba es probable que los materiales sean calco- } \\
\text { alcalinos relacionados con la Formación Paso Real }\end{array}$ \\
\hline Comején & $\begin{array}{l}\text { Facies turbidíticas (areniscas) (Formación Térraba, } \\
\text { Unidad Lagarto). Conglomerados areniscas y lutitas. } \\
\text { Mioceno superior (Formación Curré) }\end{array}$ \\
\hline San Joaquín, Calienta Tigre & $\begin{array}{l}\text { Facies turbidíticas (areniscas) (Formación Térraba, } \\
\text { Unidad Lagarto) }\end{array}$ \\
\hline $\begin{array}{l}\text { Oka, Jakyaka, Murur Bisuk, } \\
\text { Ditsiri Suk, Bekis, Santa María, } \\
\text { Sipar, Palmital }\end{array}$ & Cuerpos intrusivos (Grupo Granito-gabro talamanca) \\
\hline Ujarrás & $\begin{array}{l}\text { Cuerpos intrusivos (Grupo granito-gabro talamanca), } \\
\text { Grupo granito-gabro talamanca y abanicos, intrusivo } \\
\text { en bloques. }\end{array}$ \\
\hline Achiote & $\begin{array}{l}\text { Abanicos aluviales antiguos y jóvenes (Formación } \\
\text { Valle El General) }\end{array}$ \\
\hline Sebror, Buenos Aires & $\begin{array}{l}\text { Abanicos aluviales jóvenes (Formación Valle El } \\
\text { General) }\end{array}$ \\
\hline Salitre & $\begin{array}{l}\text { Areniscas tobáceas con intercalación de } \\
\text { conglomerados, limolitas y ocasionalmente lutitas } \\
\text { (Formación Curré) (Mioceno medio) }\end{array}$ \\
\hline
\end{tabular}

Fuente: Obando y Kussmaul, 2009; Alvarado et al., 2009.

El contexto litológico es afectado por una serie de fallas, así como por movimientos relativos de carácter horizontal. Una vez que el relieve se torna plano $\mathrm{u}$ ondulado a partir de los $400 \mathrm{~m}$, se encuentran principalmente materiales sedimentarios, correspondientes a abanicos aluviales jóvenes, pertenecientes a la Formación Valle del General (Obando y Kussmaul, 2009).

También es posible identificar diferentes escenarios topográficos, los cuales van desde áreas planas y plano-onduladas, a zonas de laderas con fuertes 
pendientes (Figura 3). En el caso de las sabanas ubicadas dentro de la cuenca del río Ceibo, estas se extienden hasta muy cerca de la división continental, cerca de los cerros Brunka (3 030 metros sobre el nivel del mar (msnm)), Akata (3002 msnm), Elí (3 $097 \mathrm{msnm}$ ), Aka (3 $225 \mathrm{msnm}$ ), Dúrika (3 $280 \mathrm{msnm}$ ) y Akó (2 $995 \mathrm{msnm}$ ) y hasta aproximadamente los $400 \mathrm{msnm}$ en las afueras de la ciudad de Buenos Aires, donde se observa una topografía plano-ondulada que desciende hasta el cauce del Río General.

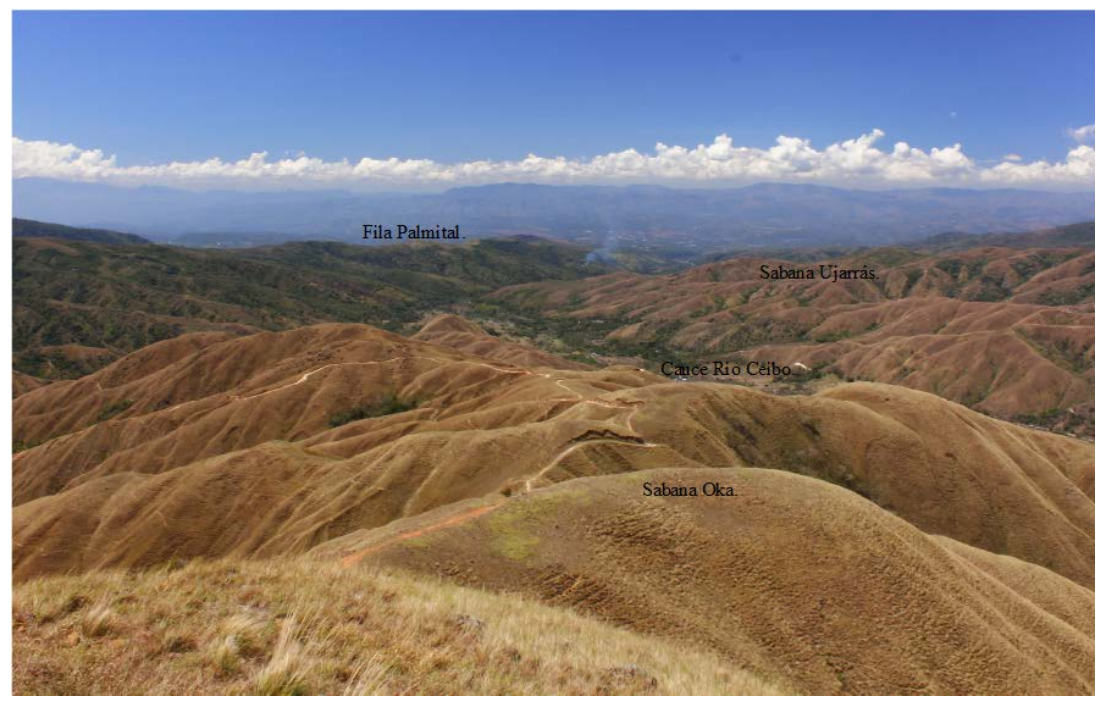

Figura 3. En primer plano: área de altas pendientes en Sabana Oka. A la derecha de la imagen el cauce del Río Ceibo y un fragmento de Sabana Ujarrás.

Fuente: Godínez, 2017.

Sobre las áreas de mayor pendiente, se puede observar un relieve de facetas triangulares y afloramiento de rocas de diferente magnitud. También, y producto de una combinación entre las características geológicas, geomorfológicas y climáticas, se observan una importante cantidad de desprendimientos en forma de cuchara e incluso importantes deslizamientos.

El contexto geomorfológico resulta de las interacciones de los materiales con factores tectónicos y climatológicos. El clima imperante en la región es tropical muy húmedo, con precipitaciones de $3450 \mathrm{~mm}$ y una temperatura promedio de $26^{\circ} \mathrm{C}$ (Gómez, 1986). La estación seca se extiende de diciembre a abril, y es muy severa pues los riachuelos de toda el área disminuyen significativamente el caudal, aunque algunos no llegan a secarse del todo, ni siquiera durante el período más intenso de la época seca. Estas condiciones, exponen a la vegetación a un importante estrés 
hídrico. Este estrés, se combina con fuertes ráfagas de viento que azotan el área de diciembre a febrero, y que están asociados a las masas de aire provenientes del norte del continente. Estos vientos, sirven como medio de dispersión de los fuegos, que anualmente afectan importantes extensiones de las sabanas.

A nivel edáfico, los suelos del área corresponden a entisoles y oxisoles, los cuales se caracterizan, en el primer caso, por un grado de desarrollo muy insipiente, y en el segundo caso, corresponden a suelos en un estadio de desarrollo muy avanzado. Ambos tipos de suelos son muy poco fértiles, y tienen una presencia variable de nutrientes. Estas características, no los hacen aptos para labores agropecuarias, salvo agricultura de subsistencia. Los suelos de las grandes sabanas de la cuenca del río Ceibo, quizás se podrían usar para el cultivo de piña a nivel industrial. Sin embargo, las características topográficas con pendientes muy pronunciadas hacen que el área no sea adecuada para este cultivo.

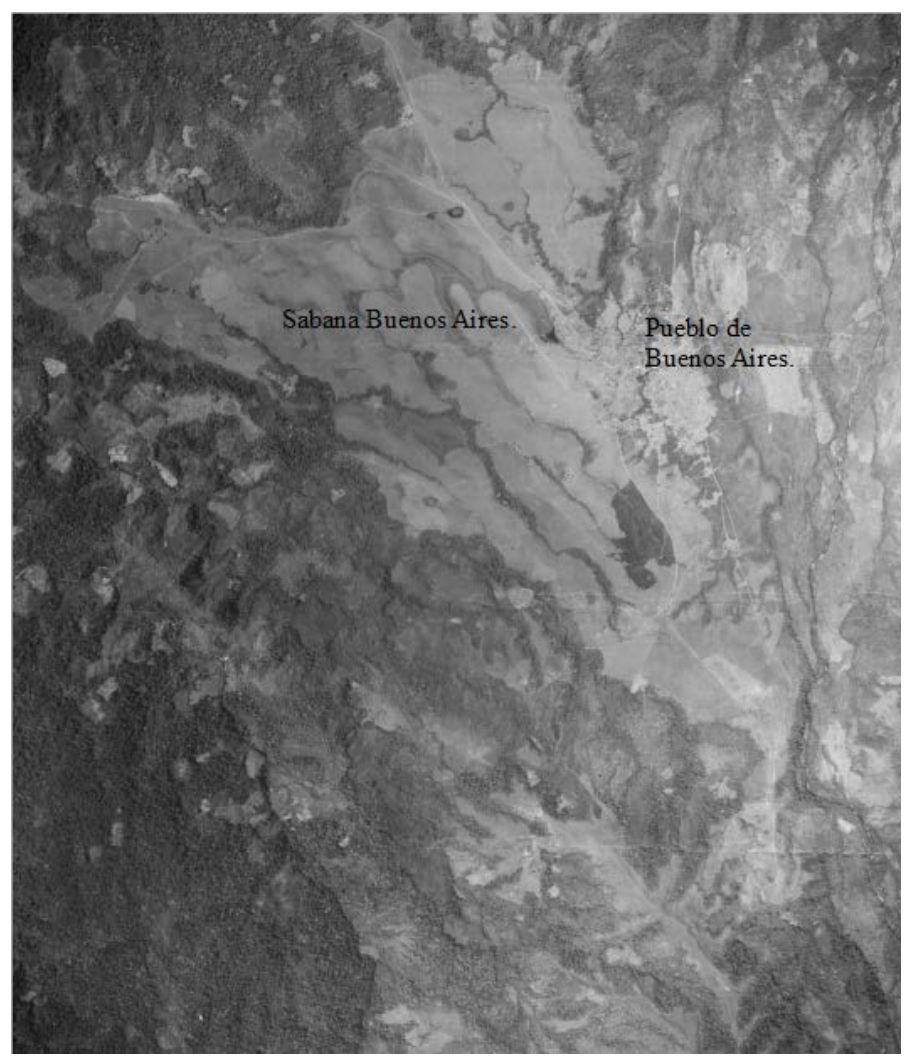

Figura 4. Sabana Buenos Aires en 1948. En el extremo derecho y al centro de la imagen se puede observar el pueblo de Buenos Aires.

Fuente: Instituto Geográfico Nacional. Fotografía aérea Monocromática. 
Los cambios en el uso de la tierra, han causado la pérdida del ecosistema. Según Gómez (1986) estos cambios son la causa de que este ecosistema esté sufriendo una alteración similar a la del bosque tropical húmedo. Las sabanas, han sido reemplazadas por actividades agropecuarias y urbanas (p.ej., las sabanas Buenos Aires, El Tigre y Sebror). La sabana de Buenos Aires prácticamente ha desaparecido, debido al desarrollo urbano del pueblo homónimo (Figura 4).

Además, el cultivo de piña ha tenido lugar en las sabanas desde 1960. Las plantaciones de piña, han provocado la pérdida de áreas de sabana, e incluso la pérdida total de algunas de estas, como por ejemplo la sabana El Tigre. El reemplazo de la sabana por áreas cultivadas de piña, tiene como límite altitudinal los $400 \mathrm{msnm}$, y está asociado a un cambio en la topografía, de área plana y planoonduladas a zonas con pendientes muy abruptas.

\section{El contexto paleoclimático y paleoecológico}

Al examinar a través del tiempo la permanencia del ecosistema de sabana en el sur de Costa Rica, inicialmente Kesel (1983) sugiere que entre 17.050 y 8.810 Antes del Presente (AP) hubo una mayor presencia de especies de bosque tropical seco, así como especies propias de la sabana, tales como Byrsonima crassifolia en la cuenca del Río General. Esta condición podría estar correlacionada, con el efecto de la última glaciación pleistocénica, así como con una menor precipitación y condiciones más áridas, las cuales se han reportado para la Cordillera de Talamanca y la costa pacífica de Panamá en el lago La Yeguada y en Monte Oscuro, un extinto volcán en la parte central de Panamá (Piperno y Jones, 2003).

Gómez (1982) menciona que las sabanas de la cuenca del Río General, podrían ser remanentes de una mayor extensión de sabanas, que ocuparon la zona hace algunos miles de años, específicamente durante la última Glaciación del Pleistoceno. Protti (1996) analizó las evidencias de este fenómeno climático en el Valle del General e identificó múltiples signos de erosión por morfo-glaciares, tales como valles en forma de $\mathrm{U}$, rocas con acanaladuras y estrías posiblemente producto del desplazamiento del hielo glaciar en la cuenca del río San Pedro. Estas observaciones están relacionadas con los hallazgos de fósiles, principalmente de mamíferos, en la localidad de San Gerardo de Limoncito, Coto Brus, relativamente cerca de las actuales sabanas. Los restos fósiles demuestran que el área fue habitada por la megafauna, principalmente especies de caballos como Protohippus gidlegi, Calippus hondurensis y Dinohippus mexicanus (Laurito y Valerio, 2016; Valerio, 2010), el armadillo gigante Scirrotherium antelucanus (Laurito y Valerio, 2016; Laurito y Valerio, 2011), y el proboscídeo Gomphotherium (Lucas y Alvarado, 2010). Todas estas especies, según los paleontólogos, debieron compartir un territorio semejante a un ambiente de sabana arbolada (Laurito y Valerio, 2010 y 2013; Valerio, 2010). De ser adecuada esta correlación, la existencia de las sabanas podría datar de 
hace 6.57 Ma (Laurito y Valerio, 2016). Además, habría evidencia de la existencia de sabanas naturales en la región sur de Costa Rica desde tiempos remotos.

Finalmente, si el poblamiento del área de sabana se remonta a 1500 años AC, tal y como lo evidencian los restos arqueológicos, los primeros pobladores debieron enfrentar un clima más seco que el presente. Según Islebe y Hooghiemstra (2005), el análisis de la laguna La Chonta demuestran condiciones más áridas alrededor del 1200 AP. Estos datos muestran un aumento de la sequía que condujo al colapso de la civilización maya entre 1300 y 1100 AP. Ante este panorama, los primeros pobladores de lo que hoy es el cantón de Buenos Aires vivieron en un ambiente de sabana, quizás arbolada, de origen natural, y que comenzó a ser alterada por el uso aborigen.

\section{El poblamiento humano pre-colombino y el origen de la sabana}

El poblamiento humano pre-colombino del territorio de lo que hoy se denomina la sub-región del Diquís se remonta a 1500-300 antes de Cristo (a.C.) (Corrales, 2001). Corrales (1985) indica que Curré es la ocupación alfarera más antigua documentada en el sur de Costa Rica, e incluso la más antigua de la región del Gran Chiriquí, y se ubica temporalmente entre el periodo Precerámico (4800-300 a.C.) y la fase Agroalfarera Aguas Buenas y Bugaba (200-600 después de Cristo (d.C.)). Rojas (2001) propone que el sitio Curré tiene más de 3000 años de evolución. Como evidencias de estos primeros pobladores agro-alfareros, existen restos de cerámica, piedras de moler y rayadores de sílex encontrados en el sitio Curré en la parte media de la cuenca del Río Térraba (Murillo y Baldí, 2002).

Estos primeros pobladores, ya contaban con herramientas para talar el bosque y para la agricultura. Entre las evidencias se encuentran hachas de piedra y cuñas (Corrales, 1985). También se han encontrado herramientas fabricadas con diferentes tipos de rocas y minerales, tales como andesitas y calcedonias correspondientes a la Fase Talamanca (4600-2300 a.C.), así como herramientas de cuarzo y obsidiana de la Fase Boquete (2300-1500 a.C.). Esto demuestra que los primeros pobladores, tenían la capacidad tecnológica de alterar el ecosistema a través de la tala del bosque, la agricultura, y el uso del fuego.

Otros sitios que también muestran evidencias claras de poblamiento, son el río Ceibo y la desembocadura de los ríos Coto Brus, Cabagra y Limón. Haberland (1961) reporta una serie de montículos alineados en las márgenes del Río Ceibo. Este autor ubica el área dentro de la fase arqueológica Boruca, con una edad de entre 1000 a 1500 (d.C.). Robert Drolet (1983) identifica una serie de aldeas y cementerios cercanos a la desembocadura de los ríos Coto Brus, Cabagra, y Limón, y que él denomina "murciélago", entre los años 1980 y 1981. Dentro de la misma región, se han encontrado una serie de montículos de hasta $2 \mathrm{~m}$ de altura en el sitio Finca Remolino (Coronado, 2006). 
Al momento de la conquista, y alrededor del año 1563 existían pueblos como Couto, Burucac, Cía, Uriaba, Xariaxaba, Yabo, Cabara, Duyba, Barezto, Tabicte, Arobara, Cabangara, Quecabangara, Cuacua, Quecura, Baricara, Curubi, Turuca y Ucacara. Estos eran pequeños asentamientos que se localizaban en las sabanas (Sánchez y Arrea, 2004). Por esto, los techos de los ranchos de estos poblados se construían con pasto de la sabana, el cual se colocaba en pequeños rollos que se amarraban a una armazón de madera que a su vez era colocada sobre soportes más gruesos que evitaban la entrada de agua. Estos techos podían durar hasta 40 años. Se utilizaban tres tipos diferentes de pastos. El pasto denominado de sabana o peludo (bshac), el de comején (shupshus) y finalmente el de palito (su). Para la construcción de un rancho se utilizan hasta 2700 rollos de pasto (Stone, 1949).

Estos grupos humanos, podrían haber colaborado en la creación de las sabanas al talar el bosque con hachas de piedra, y luego usar el fuego para eliminar el resto de la vegetación. Dentro de las leyendas transmitidas de generación en generación, están las que hacen referencia no solamente a los primeros habitantes del área, sino a cómo estos, por orden divina, crearon las sabanas y utilizaron estos espacios inicialmente para agricultura.

Dentro de las narraciones Bribris se relata que: "Los sorbLu y los Kambra hicieron las sabanas. Ellos eran de semilla indígena, como los bribris. Ellos sembraban, y donde sembraban la tierra, allí se convertía todo en zacate, no volvían a crecer árboles, ni el maíz, ni otras cosechas. Dios los mandó al mar" (Bozzolli, 1977). "KábLa, Kámbra son los primeros cultivadores de la tierra, se dice que crearon la sabana de Buenos Aires, que eran malos, que Dios los mandó al mar" (Bozzolli, 1977).

Por su parte, los Cabécares también tienen un relato que describe el origen de las sabanas:

Según ellos, antes todo era montaña, pero Sibü envió una pareja de Talamanca, la que inició el poblamiento de lo que hoy es Ujarrás y también crearon las sabanas. Estos seres eran Arabulö y Sorbulö. Ellos podían construir herramientas de piedra. Sin embargo, Arabulö no obedecía a Sibü y trabajaba de día y de noche cortando el bosque. Entonces Sibü pidió a Sorbulö que destruyera las herramientas, y les quitó el conocimiento para hacerlas. Cuando Sibü detuvo la deforestación, maldijo la tierra, y desde entonces por eso no se puede sembrar. Sibü se llevó sus especies a la montaña G. Padilla (comunicación personal, 13 de abril, 2019).

\section{El rol del fuego en las sabanas}

En 1891, según un testimonio de José Sotero Carrera Ortiz, las sabanas se extendían de forma continua desde Ujarrás hasta Helechales, y cuando se iniciaba un fuego en estas duraba hasta 15 días en apagarse (Barrantes, 2015). Contemporáneo a la fecha del testimonio anterior, Henri Pittier hizo una visita a 
Buenos Aires. En este viaje, el científico destacó la acción del agente de Policía, José Figueroa Rucavado, en la conservación del pasto natural que constituía las sabanas al afirmar:

aquel prohibía con mucho tino las quemas de las sabanas, pero, como no obstante los vecinos las justificaban diciendo que las cenizas servían de abono, Figueroa había permitido quemar después de los 25 de marzo de cada año, para que las lluvias cayeran pronto sobre el terreno y la ceniza se aprovechara y no correr el riesgo de que el viento las llevara a otros sitios (Chacón, 1986).

Las dos referencias anteriores, demuestran que el uso del fuego ha sido parte integral de la existencia de las sabanas de Buenos Aires (Figura 5). Haberland (1961) sugiere que las sabanas de Buenos Aires no representen la vegetación natural del área, y que más bien son el resultado de la acción humana a través del uso combinado de la agricultura y el fuego de una manera intensiva. Este comportamiento, de tala del bosque y desarrollo de áreas de cultivo está documentado a través del análisis de yacimientos de carbón y polen en lagos y humedales neotropicales.

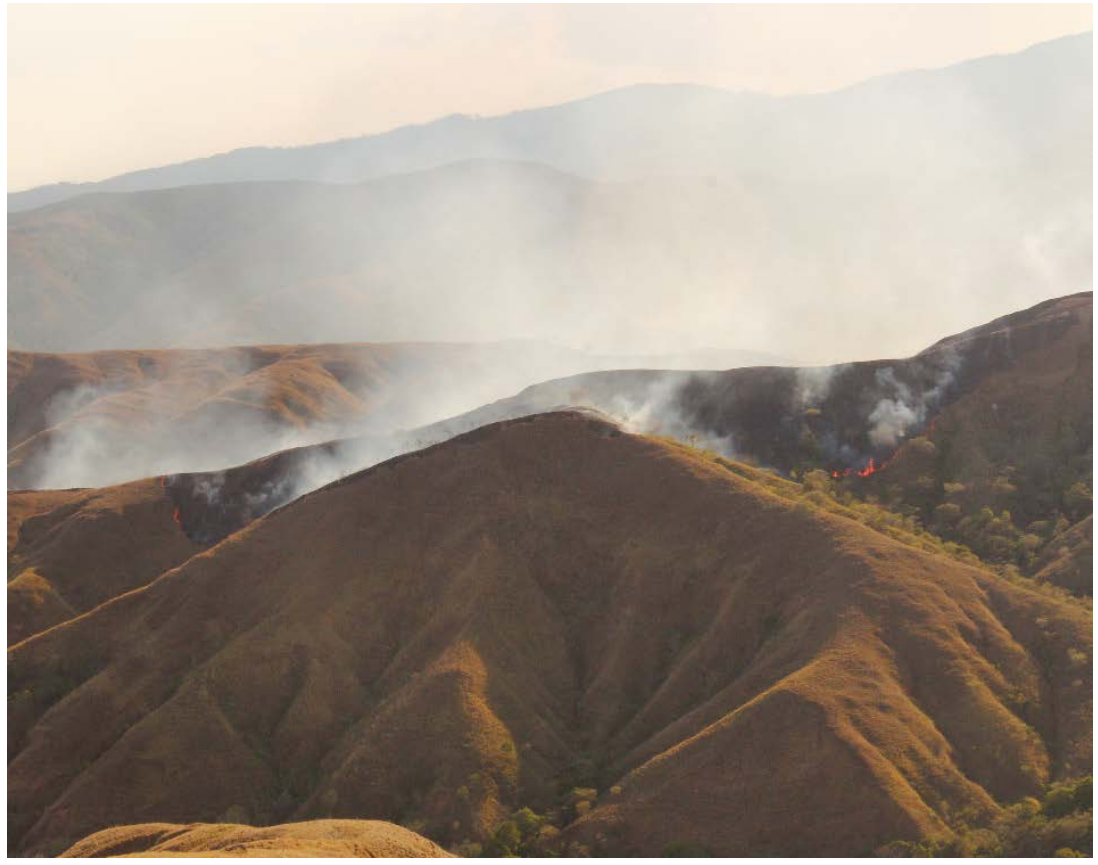

Figura 5. Fuego avanzando por sabana Bekis, marzo de 2017.

Fuente: Godínez, 2017. 
Las sabanas de Buenos Aires, sufren los embates del fuego por lo menos una vez al año, principalmente durante la época seca, en la que se da una combinación de ausencia de precipitaciones, presencia de abundante materia vegetal muy seca, y fuertes vientos. Estos factores facilitan la propagación de fuegos, que afectan amplias áreas de sabanas. También tienen lugar incendios de menor magnitud durante la época lluviosa, estos abarcan desde unos cuantos metros cuadrados hasta unas cuantas hectáreas. Para que estos fuegos sucedan, deben combinarse un par de días sin precipitaciones y temperatura elevadas, para que el material herbáceo sea susceptible de combustión (J. Ortíz, comunicación personal, 28 de junio, 2018).

\section{La exploración científica de las sabanas}

Las exploraciones científicas de las sabanas se inician con el trabajo de José María Figueroa en 1871 (Vargas, 2011). Figueroa elabora una serie de bosquejos, en los que representa el área de sabana, principalmente en Buenos Aires y Ujarrás. Además, hace referencia al uso continuo del fuego por parte de los pobladores.

En 1891, el Instituto Geográfico Nacional financia la expedición de Henri Pittier y Adolphe Tonduz a la zona sur, en donde se documenta la existencia de sabanas y se realizan las primeras identificaciones botánicas que incluyen géneros como Eragrostis, Paspalum, Digitaria, Cyperus y Rinchospora, típicamente sabaneros. Entre las especies identificadas por estos naturalistas, está Paspalum pictum, colectada en la sabana Boruca por Pittier. Tonduz colectó en los alrededores del poblado de Boruca, e identificó a Cyperus chorisanthus y Eleocharis durandii, ambas especies endémicas. Otro aporte de Tunduz, fue el descubrimiento de la especie Eleocharis sulcata (Díaz, 2015).

Carlos Wercklé (1909) en su obra titulada La Subregión fitogeográfica costarricense apunta:

En la región del Río General se encuentran, a una altura de 200 m-400 m, extensos helechales en lugares sin ninguna vegetación arbórea, donde los helechos de rizoma corredor subterráneo cubren el suelo con exclusión de toda otra vegetación formando un charral densísimo de $2 \mathrm{~m}$ a $3 \mathrm{~m}$ de altura... Unas colinas y montañas áridas están cubiertas de zacate (gramíneas) de hoja angosta y tenaces con unos pocos nances Byrsonima crassifolia, y unos pocos arbustos de "lengua de vaca", Conostegia, varias especies.

Paul Standley (1938), en su compendio de Flora de Costa Rica, muestra que la vegetación de sabana no se extiende al norte de Costa Rica, excepto en pequeñas áreas, pero que se ha desarrollado bien en Guanacaste, y en la parte sureste húmeda en Térraba y Boruca cerca del Golfo Dulce. El mismo autor indica que las sabanas son características del pacífico en Panamá. Standley identifica 
especímenes y corrobora las identificaciones realizadas por Pittier y Tonduz en 1891 (Tabla 3).

Tabla 3. Plantas identificadas por Pittier y Tonduz (1891) y Stanley (1937), en las sabanas Buenos Aires y Boruca

\begin{tabular}{ll}
\multicolumn{1}{c}{ Especie } & \multicolumn{1}{c}{ Localidad de colecta } \\
\hline Paspalum lineare & Sin datos \\
Paspalum multicaule & Sabanas Buenos Aires y Boruca \\
Paspalum pictum & Sabana Boruca (Pittier) \\
Rottboellia aurita & Sin datos \\
Sporobolus ciliatus & Sin datos \\
Sporobolus inclius & Sin datos \\
Trachypogon montufari & Sin datos \\
Cyperus chorisanthus & Bosques de Boruca. Endémica (Tonduz) \\
Eleocharis durandii & Sin dato. Endémica. (Tonduz) \\
Eleocharis interstincta & Sabana Buenos Aires \\
Eleocharis plicarhachis & Sabana Buenos Aires \\
Eleocharis sulcata & Sabana Boruca (Tonduz) \\
Rynchospora armerioides & Sabana Buenos Aires \\
Rynchospora clarkei & Sabana Buenos Aires \\
Rynchospora cyperoides & Sabana Buenos Aires \\
Rynchospora eximia & Sabana Boruca (Tonduz) \\
Rynchospora glauca & Sabanas Boruca y Buenos Aires \\
Rynchospora hirsuta & Sabana Buenos Aires \\
Rynchospora longispicata & Sabana Buenos Aires \\
Scleria lithosperma & \\
\hline Scleria macrophylla & Sin Buenos Aires \\
\hline
\end{tabular}

Fuente: Standley, 1938.

Según León (1952), la sabana es la vegetación más típica del pacífico costarricense. Este autor considera a la sabana como un tipo natural de vegetación, 
dominada por gramíneas y ciperáceas, y que ocupa espacios intermedios entre los bosques deciduos. León ubica la presencia de sabanas en Térraba, Buenos Aires, Ujarrás, Cabagra y Potrero Grande. Además, reporta que el principal uso de este ecosistema es la ganadería, la cual fue muy importante entre los siglos XVIII y XIX cuando eran administradas por misioneros franciscanos. Sin embargo, esta actividad decayó debido a la disminución del área de sabana, como resultado de las quemas repetidas las cuales convierten las sabanas en charrales poco aptos para el pastoreo. Esta idea se contrapone, a la relación ecológica positiva que parece existir entre el fugo y la vegetación de sabana. León también menciona el uso del pasto de sabana para la construcción de los techos de ranchos. El uso del pasto de sabana (Thrasya robusta) ha disminuido principalmente por la escasez de este pasto nativo.

Sandner (1962) menciona que en las sabanas de Buenos Aires se criaba ganado y que anualmente se enviaban alrededor de 1500 a 1600 reses al Valle Central. También indica que alrededor de 1908, y según datos de Pedro Pérez Zeledón y Elías Leiva, en Buenos Aires habían alrededor de 104 ranchos y 565 personas, y que en la sabana que circundaba a esta población pastaban alrededor de 1028 cabezas de ganado, 300 caballos, y 721 cerdos.

Gómez (1986) apunta que fue Hellmuth Polakowsky el primer científico en mencionar la presencia de sabanas en Costa Rica en 1879. L. Weibel y P. E. James hacen los primeros mapas de distribución de sabanas en 1939 y 1942. Para 1943, el Servicio Forestal de Estados Unidos publica "Forest types map of Costa Rica" e incluye en esta cartografía a las sabanas. Para Gómez existieron sabanas autóctonas en Costa Rica, en las cuales vivió la megafauna pleistocénica en un ambiente abierto. Sin embrago, la extinción de la megafauna impactó la permanencia natural del ecosistema, el cual se modificó aún más con la llegada de la especie humana. Según Gómez (1986) la evidencia de la colonización humana se refleja en la elevada abundancia de especies como el nance (Byrsonima crassifolia), el jícaro (Crescentia alata), y el raspahuacal (Curatella americana) que aún caracterizan a muchas de las sabanas.

Finalmente, en el 2006 el Instituto Nacional de Biodiversidad y The Nature Conservancy exploraron la sabana La Esperanza, realizando un amplio trabajo de identificación de especies botánicas. A la fecha, es la única sabana del sur del país que cuenta con un inventario intensivo. Las demás sabanas presentan listas de especies pero sin el registro geográfico de dónde fueron recolectadas las muestras.

\section{Conclusiones}

El ecosistema de sabana del sur de Costa Rica ha tenido la atención de prominentes científicos, tanto nacionales como extranjeros, de diferentes campos. Sin embargo, 
todavía falta por determinar su origen y evolución, y aclarar si tiene un origen estrictamente antropogénico o son de origen natural.

La utilización del ecosistema de sabana en el sur de Costa Rica, es de larga data. Así lo demuestran las evidencias arqueológicas. El uso habitacional, productivo e incluso funerario, es bastante patente. En las sabanas localizadas en sitios de mayor elevación, se pueden observar tumbas aborígenes, que fueron profanadas décadas atrás.

El estudio de la flora de las sabanas, ha sido un tema importante desde el inicio de la exploración científica. Sin embargo, es necesario profundizar el conocimiento de la flora de la sabana, e incluir aspectos de su estructura y distribución espacial. El efecto del fuego sobre dicho ecosistema, parece tener una muy amplia historia, que se remonta incluso a antes de la conquista, y que se proyecta hasta nuestros días. Se requiere implementar análisis temporales y espaciales, que monitoreen el impacto del fuego sobre este ecosistema. Esto reviste especial importancia, pues una parte considerable del área de las sabanas es sometida a fuegos provocados cada año.

Finalmente, ante un escenario de cambio climático como el que se prevé, con baja precipitación y altas temperaturas, podría afectar la distribución, estructura, y composición de especies de estas sabanas. Se presume que el proceso de calentamiento, podría extender el área de sabanas en el futuro cercano, por lo que es de considerable importancia comprender la interacción entre comunidades con Buenos Aires de Puntarenas y el ecosistema sabana presente es su territorio.

\section{Referencias}

Alvarado, G., Barquero, R., Taylor, W., López, A., Cerdas, A. y Murillo, J. (2009). Geología de la Hoja General. Revista Geológica de América Central, 40, 99-109. https://www.doi.org/10.15517/RGAC.V0140.4189

Artavia, L. (2011). Las sabanas húmedas del Sur de Costa Rica, una Caracterización Fitogeográfica. Revista Geográfica de América Central, 2(47E).

Barrantes, C. (2015). Lejano Diquis. Costa Rica: Editorial Universidad Estatal a Distancia (UNED) y Editorial Instituto Costarricense de Enseñanza Radiofónica (ICER).

Beerling, D. y Osborne, C. (2006). The origin of the savanna biome. Global Change Biology, 12, 2023-2031. https://www.doi.org/10.1111/j.1365-2486.2006.01239.

Bozzolli, M. (1977). Narraciones Bribris. Revista Vínculos, 3(1-2), 67-104.

Calabrese, J., Vazquez, F., López, C., San Miguel, M. y Grimm, V. (2010). The independent and interactive effects of tree- tree establishment competition and fire on savanna structure and dynamics. The American Naturalist, 175(3), 44-65.

https://www.doi.org/10.1086/650368.

Caylor, K. y Shugart, H. (2006). Pattern and process in savanna ecosystems. En D Odorico, P., Runyan, C. y Porporato, A. Dryland Ecohydrology, 259-281, Netherlands: Springer. 
Corrales, F. (1985). Prospección y excavaciones en el sitio Curre (P-62-Cé), Valle del Diquis, Costa Rica. Revista Vínculos, 11(1-2), 1-18.

Corrales, F. (2001). Los Primeros Costarricenses. Costa Rica: Editorial Nuestra Tierra.

Coronado, C. 2006. Evaluación del rasgo del sector arquitectónico N. VI del asentamiento Los Altos P-655 LA (Cat-UCR-517), Potrero Grande, Buenos Aires, Puntarenas. Tesis de Licenciatura. Escuela de Antropología. Universidad de Costa Rica, San José.

Chacón, L. (1986). Buenos Aires, cantón de Puntarenas: apuntes para su historia. Revista del Archivo Nacional, 44-50(1-12), 5-166.

Díaz, R. (2015). La transformación de las sabanas de Buenos Aires de Puntarenas, Costa Rica (1870-2010). En Lértora, C. (coord.), Sustentabilidad y conservación. Proyecto Ecoepisteme. (pp. 109-137), Argentina: Fundación FEPAI.

Drolet, R. (1983). Al otro lado de Chirriquí, El Diquís: Nuevos datos para la integración cultural de la región Gran Chirriquí. Revista Vínculos, 9(1-2), 25-76.

Godínez, I. (2017). Trabajo de campo. San Vicente de Ujarrás, Buenos Aires, Puntarenas, Costa Rica.

Gómez, L. (1986). Vegetación de Costa Rica: apuntes para una biogeografía costarricense. Costa Rica: Editorial Universidad Estatal a Distancia.

Haberland, W. (1961). Arqueología del Valle del Río Ceiba. Buenos Aires. Informe semestral (enero-junio). Costa Rica: Instituto Geográfico Nacional.

Harris, D. (1980). Tropical savanna environments: Defination, distribution, diversity, and development. Human Ecology in Savanna Environments.

Higgins, S., Bond, W., February, E., Bronn A., Euston, D., Enslin, B., Govender, N., Rademan, L., O’Regan, S., Potgieter, A., Scheiter, S., Sowry, R., Trollope, L. y Trollope, W. (2007). Effects of four decades of fire manipulation on woody vegetation structure in savanna. Ecology, 88(5), 1119-1125. https://www.doi.org/10.1890/06-1664.

Islebe, G y Hooghiemstra, H. (2005). Historia del clima y de la vegetación montañosa de Costa Rica desde el último glaciar. En: Kappelle, M. y Horn, S. Páramos de Costa Rica (pp. 215-236). Costa Rica: INBIO.

https://www.doi.org/10.1659/0276-4741(2007)27[95:PDCR]2.0.CO.

Kesel, R. (1983). Quaternary History of the Río General Valley, Costa Rica. National Geographic Society Research Report, 15, 339-358.

Laurito, C. y Valerio, A. (2010). Los Caballos Fósiles de la Formación Curré, Cantón de Coto Brus. Costa Rica. San José, Costa Rica: Museo Nacional de Costa Rica.

Laurito, C. y Valerio, A. (2011). Scirrotherium antelucanus, una nueva especie de Pampatheriidae (Mammalia, Xenartthra, Cingulata) del Mioceno superior de Costa Rica, América Central. Revista Geológica de América Central, 49, 45-62. https://www.doi.org/10.15517/RGAC.V0149.13101

Laurito, C. y Valerio, A. (2016). Camellos Láminos del Mioeceno tardío (Henfiliano temprano) de la Formación Curré, San Gerardo de Limoncito, Cantón de Coto Brus, Provincia de Puntarenas, Costa Rica. Revista Geológica de América Central, 54, 7-55. https://www.doi.org/10.15517/RGAC.V5410.21148

León, J. (1952). Nueva Geografía de Costa Rica. Costa Rica: Editorial Libreria La Española.

Lomolino, M., Riddle, B. y Brown, J. (2006). Biogeography. Massachusetts, Estados Unidos: Sinauer Associates, Inc. 
Lucas, S. y Alvarado, G. (2010). Fossil Proboscidea from the Upper Cenozoic of Central America: Taxonomy, Evolutionary and Paleobiogeographic Significance. Revista Geológica de América Central, 42, 9-36. https://www.doi.org/10.15517/RGAC.V0142.4169

Molina, C. (2005). Y las mulas no durmieron. Los arrieros en Costa Rica: siglos XVI al XIX. Costa Rica: Editorial Universidad Estatal a Distancia (EUNED).

Murillo, M. y Baldí, N. (2002). Informe Final Práctica de Investigación en Arqueología Sitio Brusmalis. Escuela de Antropología, Universidad de Costa RicaCosta Rica.

Obando, L. y Kussmaul, S. (2009). Geología de la Hoja Buenos Aires, Costa Rica. Revista Geológica de América Central, 41, 123-136.

https://www.doi.org/10.15517/RGAC.V0141.4184

Ortiz, J. Comunicación personal, 28 de junio, 2018. San Vicente de Ujarras. Buenos Aires. Puntarenas. Costa Rica.

Padilla, G. (2019). El origen de las sabanas según la tradición Cabecar. Comunicación personal. Con base en los relatos de su abuela materna Antonieta Beita Obando y su tio abuelo materno Luis Beita Obando. San Vicente de Ujarras. Buenos Aires. Puntarenas. Costa Rica.

Piperno, D. y Jones, J. (2003). Paleoecological and archaeological implications of a Late Pleistocene/Early Holocene record of vegetation and climate from the Pacific coastal plain of Panama. Cuaternary Research, 59, 79-87. https://www.doi.org/10.1016/S0033-5894 (02) 00021-2.

Protti, R. (1996). Evidencias de glaciación en el Valle del General (Costa Rica) durante el Pleistoceno tardío. Revista Geológica de América Central, (19-20), 75-85. https://www.doi.org/10.15517/RGAC.VOl19-20.8687

Rojas, C. (2001). Nombrando el territorio Brunca: Topónimos en legua Boruca. Revista Vínculos, 26(1-2), 1-18.

Sánchez, M. y Arrea, F. (2004). El potencial arqueológico del Golfo Dulce, Pacífico Sur de Costa Rica. Informe de Investigación. Universidad de Costa Rica, Costa Rica.

Sandner, G. (1962). La Colonización Agrícola de Costa Rica. Tomo I. Costa Rica: Instituto Geográfico de Costa Rica.

Standley, P. (1938). Flora de Costa Rica. Costa Rica: Imprenta del Museo Nacional. San José.

Stone, D. (1949). Los Borucas de Costa Rica. Costa Rica, Traducción: Editorial Imprenta Nacional. San José.

Valerio, A. (2010). Paleontología, bioestratigrafía y paleoecología de los caballos fósiles de la Formación Curré en el cantón de Coto Brus, Costa Rica. Tesis de Licenciatura. Escuela de Geología. Universidad de Costa Rica, San José.

Van Der Hammen, T. (1983). Tropical Savannas, The Palaeocology and Palaeogeography of Savannas. Bourliére Ed. Amsterdam.

Vargas, G. (2005). Sabana arbustiva del Parque Nacional Santa Rosa, Guanacaste, 1995-2004. Cuadernos de Estudios Mesoamericanos, 2. Heredia, Costa Rica: CEMEDE-UNA.

Vargas, G. (2011). La cartografía y el paisaje geográfico en el Álbum de Figueroa. En El Álbum de Figueroa: un viaje por las páginas del tiempo. San José. Costa Rica. Editorial Tecnológica. Costa Rica.

Wercklé, C. (1909). La SUBREGION Fitogeográfica Costarricense. Costa Rica: Tipografía Nacional. 